\title{
Yod
}

Revue des études hébraïques et juives

19 | 2014

Aharon Appelfeld, cinquante ans d'écriture

\section{Histoire d'une vie, Histoire de silences : une poétique de la mémoire}

The Story of a Life, Story of Silences: Poetics of Memory

$$
\text { “סיפור חיים”,סיפור של שתיקות :על הפואטיקה של הזכרון }
$$

\section{Danièle Sabbah}

\section{(2) OpenEdition}

Journals

Édition électronique

URL : https://journals.openedition.org/yod/2148

DOI : $10.4000 /$ yod. 2148

ISSN : 2261-0200

Éditeur

INALCO

Édition imprimée

Date de publication : 30 mai 2014

ISBN : 978-2-85831-214-6

ISSN : 0338-9316

Référence électronique

Danièle Sabbah, « Histoire d'une vie, Histoire de silences : une poétique de la mémoire », Yod [En ligne], 19 | 2014, mis en ligne le 16 avril 2014, consulté le 08 juillet 2021. URL : http://

journals.openedition.org/yod/2148; DOI : https://doi.org/10.4000/yod.2148

Ce document a été généré automatiquement le 8 juillet 2021.

\section{(c) (†) 8}

Yod est mis à disposition selon les termes de la Licence Creative Commons Attribution - Pas d'Utilisation Commerciale 4.0 International. 


\section{Histoire d'une vie, Histoire de silences : une poétique de la mémoire}

The Story of a Life, Story of Silences: Poetics of Memory

“סיפור חיים”, סיפור של שתיקות :על הפואטיקה של הזכרון

\section{Danièle Sabbah}

\section{NOTE DE L'AUTEUR}

Le titre de cet article fait référence à Aharon Appelfeld (2004). Histoire d'une vie, traduit de l'hébreu par Valérie Zenatti, Paris, Éditions de L'Olivier ; Paris, Seuil, coll. « Points », 2005 [publication en hébreu : Sippur hayyim, Jérusalem, Keter, 1999].

« Garder le silence, c'est ce que à notre insu nous voulons tous, écrivant. »

Maurice Blanchot, L'écriture du désastre. « Les mots bousculent tout, veulent, à tour de rôle, convaincre. Le vrai dialogue humain, celui des mains, des prunelles, est un dialogue silencieux. »

Edmond Jabès, Le Livre des Questions ${ }^{1}$.

1 Aharon Appelfeld est né en 1932, Raymond Federman en 1928, Georges Perec en 1936. Tous trois ont été enfants dans la guerre et ont subi « l'Histoire avec sa grande hache ${ }^{2}$, dans laquelle leurs parents ont disparu à jamais...

2 Pour ces enfants qui ont vécu solitairement le traumatisme de la guerre, pour qui les événements n'ont pas été verbalisés, que reste-t-il de cette période? Des sensations? De vagues et brutales visions? Comment les faire revenir, et comment les écrire, 
d'autant que cette période pose par elle-même de redoutables problèmes de représentation?

Devenu écrivain, chacun d'entre eux a élaboré une forme originale pour répondre à l'impossibilité d'un récit qu'ils s'obstinent à faire, malgré tout. Concernant Aharon Appelfeld, je voudrais montrer que son invention littéraire repose grandement sur le silence comme force agissante de la narration. Racontant l'histoire d'une vie - parmi d'autres -, il introduit un acteur peu courant, généralement confiné au rôle de faire valoir de la parole : le silence. Il le déploie, le fait résonner, en décline les significations et en fait une véritable langue tout aussi riche et polysémique que la parole.

Plus précisément, nous verrons que le silence accompagne le narrateur dans les différentes étapes de sa vie. D'une ambivalence profonde, tantôt remède, tantôt poison, pour le jeune enfant, le silence devient, après la guerre, pour le jeune homme confronté à un nouveau monde auquel il est contraint de s'adapter, source de restauration d'une identité blessée. Par la fonction symbolique ainsi dévolue au silence, se trouve déplacée la caractéristique générique que le titre laisse entendre. Loin de se réduire à une autobiographie ${ }^{3}$, Histoire d'une vie relèverait plutôt du " roman d'apprentissage », du fait que les sensations premières contiennent en germe les fondements d'une vie, ce qui appert au moment du passage à l'écriture, lequel n'est pas sans proximité avec ce grand roman de la mémoire qu'est le roman proustien, comme j'essaierai de le pointer. Mais cela se limiterait à quelque problématique littéraire autour d'un «tragique épisode ", si l'écrivain n'avait la conviction que l'Anéantissement a fait voler en éclats le monde ancien et que cela retentit jusque dans la nécessité de créer des «formes nouvelles »". En réalité, par son art de l'ellipse et de la discrétion, Aharon Appelfeld invente un genre, dans le domaine délicat et controversé du témoignage, un genre de type oxymorique, celui du témoignage poétique.

\section{Le silence comme «pharmakon »: Silere et tacere}

5 Ce que nous apprend l'auteur de Histoire d'une vie, au rebours d'une société gouvernée par la parole ( Au commencement était le Verbe ${ }^{5}$...) et contrairement à l'art prétendu de la « conversation », c'est que le silence n'est pas pause entre les paroles, il n'est pas l'envers du verbe, le fond neutre qu'il s'agirait de meubler. Le silence n'est ni univoque ni « mono-tone » : selon les contextes, il résonne de timbres différents, sa tessiture est nuancée par l'étendue du blanc dont émergent les mots et à laquelle ces derniers retournent, sitôt énoncés.

Du fait que le silence renvoie aux deux limites extrêmes de la parole (son avant et son après), il revêt au moins deux significations radicalement opposées : à un de ses pôles, il est l'avant-langage où tout fait signe - les mots, les gestes et les intonations - pour un corps en éveil qui capte les sensations et les gestes bienveillants ou hostiles, il est langage pour l'infans ${ }^{6}$ qui ne peut parler, tandis que, à l'autre extrémité, se trouve le silence des situations où l'humain régresse à une sauvagerie taciturne, il est le nonlangage de l'homme livré à sa bestialité et annonciateur d'un péril extrême. Ajoutons à cela que, pour un même sujet parlant, tantôt il peut être le signe de la complétude, l'audelà des mots - échange amical et chaleureux ou recueillement intérieur pour une écoute profonde -, tantôt, inversement, il peut être le mutisme de celui qui retient ses propos quand ceux-ci portent la mort en eux. Enfin, l'après-guerre produit un autre 
silence, celui, terrible, des victimes qui ne peuvent parler ou à qui on impose de se taire pour que « la vie continue».

7 Cette ambivalence du silence que le récit d'Appelfeld révèle, cette capacité de retournement, tantôt bulle bienfaisante, tantôt méfait mortel, n'est pas sans évoquer la notion grecque de "pharmakon ", cette entité à double face, tantôt remède et tantôt poison. Dans le Phèdre de Platon - et dans le commentaire qu'en donne Jacques Derrida $^{7}-$, le "pharmakon » s'applique à l'écriture, invention aussi ingénieuse que risquée, qui d'un côté permet la mémoration, mais qui d'un autre côté, détruit la mémoire vive, le cheminement dialectique vers la mémoire et la vérité. Même ambivalence chez Appelfeld, concernant le silence, même capacité de retournement du signe : tantôt il est silence heureux, signe d'une harmonie, d'un échange serein, havre vers lequel se réfugier quand la pression extérieure est trop forte, tantôt il est silence angoissé, avertissement d'un danger mortel. Il faut ici ajouter que, tant sur le plan de la narration que sur celui de l'apprentissage que fait l'enfant, l'un prépare l'autre, le bon silence se retournera en silence destructeur. Quant au plan du passage à l'écriture, le silence se révèlera également «pharmakon » du fait qu'il est à la fois et l'obstacle au récit et sa solution.

\section{Silence heureux de la contemplation}

8 La version heureuse du silence, c'est celle du premier temps de l'enfance. De sa petite enfance, de ce bonheur rassurant que l'enfant entouré de soins a connu, ce qui lui revient est d'abord le silence.

Le narrateur enfant, plus sensible aux atmosphères qu'aux discours, aux émotions qu'aux propos qui les décrivent, capte la «musique» des relations entre adultes. Il apprend auprès des êtres sereins et aimants de sa famille le silence tranquille et plein qui est leur mode de communication et il en savoure les infimes variations de timbre: silence de la complétude, du recueillement pieux, de l'intériorité sensible. Dans ce contexte, la parole est plutôt signe de dispersion, de futilité, de fébrilité, c'est le cas pour l'enfant qui parle, qui questionne, qui s'inquiète... et qui finit par apprendre auprès des siens à faire silence, pour s'initier à lire d'autres signes.

10 Le dialogue silencieux, c'est en premier lieu auprès de sa mère que le petit Erwin l'apprend, en goûtant la quiétude heureuse d'une mère contemplative et sensible. Au cours des promenades, lors des longues soirées à la campagne, chez ses parents ou chez l'oncle Félix, elle contemple le paysage, se met à l'écoute des bruits, se laisse pénétrer par la nature. À la maison également, c'est le silence qui l'emporte, un silence qui se passe de parole parce qu'il exprime un trop plein de tendresse :

À la maison aussi le silence est plus prégnant que la parole. De ces jours lointains et enfouis il ne reste aucune parole dans ma mémoire, seulement les regards de ma mère. Ils contenaient tant de douceur et d'attention à mon égard que je les sens aujourd'hui encore ${ }^{8}$.

11 Plus encore que sa mère, le père de sa mère est un homme silencieux et l'enfant le remarque avec insistance :

Il ne parle presque pas. [...] Grand-père nous impose à tous le silence. [...] Grandpère marche sans rien dire, mais son silence n'est pas effrayant. [...] Grand-père demeure silencieux, ce qui s'échappe de sa bouche est comme ravalé et incompréhensible? . 
12 La paix de cet homme est fondée sur une foi tranquille qui s'énonce simplement : « Dieu est dans les cieux et il n'y a pas lieu d'avoir peur $»^{10}$. Silence également ou plutôt, murmure pieux des hommes à la synagogue chez qui l'enfant voit, lorsqu'ils ôtent leur châle de prière, qu' « un étonnement silencieux brille dans leurs yeux » ${ }^{11}$. Auprès d'eux, l'enfant se fait plus recueilli, plus réceptif: "Moi aussi, j'ai appris à me taire et à écouter les sons légers qui m'entourent $»^{12}$.

L'oncle Félix lui fait découvrir un silence différent, celui qui bruisse de la vie de la nature, des oiseaux, des loups : « Le silence de la maison de l'oncle Félix avait un timbre particulier. Il était naturel et vous enveloppait d'une agréable sollicitude ${ }^{13}$. La maison de l'oncle Félix lui offre la propédeutique d'un monde autre, le monde de la nature auquel la guerre le condamnera un peu plus tard, quand, errant dans la forêt, il se mettra à l'écoute des bruits de la vie animale, et le monde de ce petit Ruthène que l'oncle a adopté, mais qui s'est enfui pour retrouver sa mère démente : «Je m'identifiais à lui et j'imaginais sa fuite de la maison vers la masure maternelle ${ }^{14}$. Prémonition ? De fait, quand la guerre aura éclaté, le narrateur égaré, trempé par la pluie, va se retrouver dans la chaumière d'une femme ukrainienne, cette Maria à l'humeur instable qui l'héberge, reçoit des hommes la nuit, boit immodérément de la vodka et dont les accès de fureur finissent par se retourner contre le jeune garçon.

Lui-même est par nature un enfant solitaire, rêveur, délicat, qui souffre à l'école de la brutalité de ses congénères et se protège de ce "troupeau sauvage ${ }^{15}$ en les observant, à la récréation, depuis la fenêtre, comme il se postera plus tard à la lisière de la forêt pour regarder le monde des hommes livrés à leurs pulsions brutales ${ }^{16}$.

Cet apprentissage précoce du silence va lui être un puissant remède quand, après s'être sauvé du camp, menacé par la haine des hommes, l'orphelin se cache dans les forêts d'Ukraine. Car il retrouve alors l'état de rêverie contemplative qu'il a pratiqué auprès des siens. Le silence lui permet ainsi d'oublier l'hostilité du monde et d'entretenir à l'intérieur de lui-même le monde familier détruit par la guerre :

Je restais des heures assis dans la forêt, à contempler la flore, ou près d'un ruisseau à suivre des yeux son cours. La contemplation me faisait oublier la faim et la peur, et des visions de la maison me revenaient. [...] Ces heures de grâce me protégèrent de l'anéantissement spirituel ${ }^{17}$.

16 Cette solitude avec lui-même lui permet de renouer le fil qui le relie à sa vie antérieure d'enfant protégé, de restaurer son identité par-delà l'isolement que lui impose le monde extérieur: il illustre de fait l'opposition entre solitude et désolation que conceptualise Hannah Arendt dans sa réflexion sur le totalitarisme, l'une étant dialogue bienfaisant avec soi-même, l'autre, sentiment désespéré d'un abandon ${ }^{18}$.

17 Très tôt, l'enfant fait ainsi l'expérience de ce que Marcel Proust redécouvre au soir de sa vie : la permanence des sensations premières constitue notre identité profonde, mais elle n'est accessible qu'au prix de la méditation avec soi-même. C'est ainsi que le narrateur âgé de La Recherche du temps perdu, parce qu'il se replie dans le silence de l'écriture, peut enfin réentendre les sanglots de son enfance :

En réalité, ils [les sanglots] n'ont jamais cessé ; et c'est seulement parce que la vie se tait maintenant davantage autour de moi que je les entends de nouveau, comme ces cloches de couvents que couvrent si bien les bruits de la ville pendant le jour qu'on les croirait arrêtées, mais qui se remettent à sonner dans le silence du soir ${ }^{19}$.

Si le narrateur de La Recherche n'a pas toujours entendu les chagrins de l'enfance, c'est que les mondanités l'en ont détourné, mais pour le jeune Erwin terré dans les forêts, 
séparé du monde extérieur par l'espace magique que découpe la forêt, le bruit de la haine des hommes ne l'atteint plus et il peut laisser remonter en lui les sensations, les images, sa vie d'avant la guerre.

Ce murmure recueilli que l'enfant solitaire entretient va jusqu'à lui procurer des visions salvatrices. Dans ce temple intérieur qu'il habite, sa mère lui apparait, telle une "visitation ». Cette grâce a lieu dans un moment de bien-être inespéré. Quand, après s'être sauvé, il est entré dans la forêt qui le protège, il a pu s'y endormir, il a trouvé un pommier, il a mangé et il a bu. Une fois son angoisse et son corps apaisés, la vision se produit :

L'eau dessilla mes yeux et je vis ma mère, qui avait disparu depuis longtemps. Je la vis tout d'abord debout près de la fenêtre, en contemplation, comme elle en avait l'habitude, mais soudain elle tourna son visage vers moi, étonnée que je fusse seul dans la forêt ${ }^{20}$.

Cette présence tutélaire à ses côtés, ce dialogue muet avec ses parents, cette foi tenace en eux est sa plus sûre protection: "J'espérais sans relâche que mes parents viendraient me chercher...", dit-il ${ }^{21}$. Isolé dans cette forêt, il n'est pas livré au désœuvrement destructeur, car il a un but, retrouver ses parents : « Il me semblait que, si je trouvais le bon chemin, il me conduirait droit vers mes parents. La pensée que mes parents m'attendaient m'a protégé durant toute la guerre $»^{22}$.

21 Ainsi se trace le fil d'un silence heureux, paisible, celui de cette « langue du silence $»^{23} \mathrm{si}$ bruissante de compagnie qui était celle du grand-père, celui de la famille reconstituée en imagination.

\section{Silence tragique des temps haineux}

Toutefois, à ce silence paisible, s'oppose un tout autre silence, plus angoissant, celui des paroles rentrées, lorsque, à partir de 1938, le piège s'est refermé sur les familles juives de Roumanie et que naissent des disputes entre ses parents, que les poings se serrent d'impuissance rageuse ${ }^{24}$.

À partir de là, le silere ("faire silence ») des temps heureux s'oppose au tacere («se taire ») des temps de la haine, le silence se retourne en menace et, de philtre bienfaisant, il devient vénéneux. De même, tous les signes s'inversent: les lieux explorés aux temps heureux deviennent, pour l'enfant orphelin, les lieux de l'errance, de la peur et de l'abandon. C'est ainsi que la forêt claire avec ses maisons abandonnées, qu'il traverse avec son grand-père au retour de la synagogue ${ }^{25}$, ou les champs de maïs qu'ils admirent ensemble du haut d'une colline ${ }^{26}$, deviennent pendant la guerre les lieux mêmes de la fuite et de la persécution, et que les promenades nocturnes en famille près du ruisseau ${ }^{27}$ se retournent en marches épuisantes avec les déportés dans la boue épaisse du printemps ukrainien, puis en marches solitaires le long de cours d'eau inconnus.

Le retournement du silence en poison mortel, c'est, dans son paroxysme, le silence tragique de l'abandon du monde, celui du meurtre qui s'accomplit dans l'hostilité, la complicité ou l'indifférence. Et pour l'enfant menacé, c'est celui qu'il s'impose afin d'écouter d'où vient le danger et de taire son identité.

Lorsqu'il repère une présence humaine, sa première réaction est d'écouter le degré de menace des maisons : 
Avant de m'approcher d'une maison, d'une étable ou d'un tas de paille, je me baissais et j'écoutais, parfois des heures. D'après les bruits, je savais s'il y avait des gens et combien. Les gens étaient toujours un signe de danger. J'ai passé une grande partie de la guerre étendu sur le sol, à l'écoute ${ }^{28}$. l'impuissance de celui qui est rattrapé par le danger mortel qui le poursuit, ainsi que le silence qui l'enveloppe, ou plutôt le bruitage, ce non-langage des accès de sauvagerie : "J'entendis dans le lointain des voix étouffées, un mélange de bruits du vent et de voix humaines $\aleph^{36}$. Cris de haine des uns auxquels répondent les cris suppliants de l'enfant: depuis son poste d'observation, c'est à son propre sort, s'il venait à être repéré, qu'assiste l'enfant horrifié, dans une scène de cinéma muet. 


\section{Après la guerre, la " catharsis du silence » ${ }^{37}$}

31 Ainsi, avec la guerre, l'expérience que fait cet être, à peine sorti de l'enfance, est celle d'un retour à un stade antérieur, celui du non-verbal. Son identité se construit dans l'écoute des sensations du corps et sa capacité à les relier à ses souvenirs, à réactiver et développer ces derniers. Privé des siens, ramené à une solitude d'enfant sauvage préoccupé de sa survie, il se tait, nous l'avons vu, soit qu'il se méfie de ses rencontres, soit qu'il se trouve écrasé par la peur, soit encore que ce silence lui procure la seule manière de retrouver ses temps heureux, les langues de sa famille. De la même manière, après la guerre, dans un environnement idéologique qui écrase sa singularité, le silence est pour lui repli salutaire, purgation, catharsis. Il va lui permettre après de nombreux détours, à la fin de son apprentissage - qui correspond avec ses débuts d'écrivain -, de retrouver la mémoire du corps, son identité mise en péril et enfin ses langues, dont la plus importante, la langue de sa mère, au moment où le silence parvient enfin à déboucher sur l'écriture.

\section{La mémoire du corps}

C'est dans le silence qu'il peut espérer retrouver une mémoire "animale », celle des perceptions reçues au niveau du corps. Car c'est à un "petit animal » qu'il compare l'enfant qu'il a été, privé de langage, privé d'une société humaine aimante, préoccupé de sa survie élémentaire. La formule est récurrente :

«Durant la guerre, je ne fus pas moi. Je ressemblais à un petit animal qui possède un terrier ou, plus exactement, plusieurs terriers $»^{38}$,

ou «L'animal qui était en moi revenait m'envelopper de sa fourrure ${ }^{39}$,

ou encore "Je ressemblais plutôt à un petit animal qui aurait trouvé un refuge

temporaire dans un abri d'infortune, se nourrissant de ce que l'instant lui proposait $»^{40}$.

Et d'ailleurs, dans cette guerre où les adultes se montrent sans pitié envers les enfants, où ils les piétinent, où ils se muent brutalement en délateurs ou en assassins, c'est auprès des animaux - des jeunes animaux, ceux qui ne sont pas encore dressés à servir la haine des hommes -, qu'il trouve du réconfort, avec eux qu'il partage les besoins premiers du corps. Sécurisants, chauds, sans mauvaise surprise, ils l'accueillent et l'apaisent :

Parfois il me semble que ce ne sont pas des hommes qui m'ont sauvé, mais des animaux qui s'étaient trouvés sur mon chemin. Les heures passées auprès de chiots, de chats ou de moutons furent les plus belles heures de la guerre. Je me serrais contre eux jusqu'à en oublier qui j'étais, m'endormais près d'eux, et mon sommeil était alors paisible et profond, comme dans le lit de mes parents ${ }^{41}$.

Là où les hommes ont renoncé à se comporter comme des êtres humains, ce sont les animaux qui font son apprentissage :

Les gens étaient toujours un signe de danger. J'ai appris entre autres à écouter les oiseaux. Ce sont de merveilleux augures, pour signaler non seulement les pluies qui approchent, mais aussi les gens mauvais et les prédateurs ${ }^{42}$.

C'est pourquoi la mémoire de cette période n'est pas intellectuelle, c'est une mémoire du corps. Il ne l'a pas verbalisée, elle n'a pas été reformulée par d'autres, ce qui généralement permet à des enfants d'identifier le souvenir; court-circuitant le langage ${ }^{43}$, elle s'est inscrite dans ses sensations: «Tout ce qui s'est passé s'est inscrit 
dans les cellules du corps et non dans la mémoire $»^{44}$. S'il arrive que des visions ou des images surgissent fugacement, elles sont peu de chose auprès des souvenirs que garde chacune des parties du corps :

Parfois, des profondeurs du brouillard épais, émergent un corps sombre, une main noircie, une chaussure dont il ne reste que des lambeaux. Ces images, parfois aussi violentes qu'un coup de feu, disparaissent aussitôt [...]. Ceci concerne le domaine du conscient, mais les paumes des mains, le dos et les genoux se souviennent plus que la mémoire ${ }^{45}$.

Ce que ses cellules ont enregistré, ce sont des $"$...sensations physiques très fortes ${ }^{46}$, celles des besoins du corps, la faim, le pain, le sommeil, la peur, la recherche d'une lisière où se fondre. Et de fait, ses sens ont enregistré non des faits, des événements dûment répertoriés ou des causalités, mais des détails : "Je me souviens de milliers de détails $»^{47}$. Appelfeld insiste : pour les enfants, les souvenirs ne passent pas, comme pour les adultes, par le filtre du langage, par l'énoncé de traits communicables et tout compte fait réducteurs, par des souvenirs identifiés. La mémoire est pour eux un réservoir abondant et changeant ${ }^{48}$, les souvenirs passent par des sensations intimes qui se sont inscrites simultanément et qui, à peine retrouvées, évoquent immédiatement des fragments du passé. L'odeur de l'herbe, le toucher humide, le bruit, certaines attitudes font ressurgir des besoins (de nourriture), des douleurs (le pied qui écrase sa taille), des angoisses (un espace trop à découvert) ou des images (une foule dans une gare qui supplie pour obtenir de l'eau $)^{49}$.

Telle la madeleine pour le narrateur proustien, il suffit d'une sensation pour faire surgir des temps que le rescapé croyait perdus. L'on sait que chez Proust, la sensation délicieuse surgie inopinément recrée tout l'univers de l'enfance autour de sa tante Léonie et du village de Combray ${ }^{50}$. Elle produit un moment de temps suspendu où l'adulte retrouve intactes ses émotions premières, une mémoire enfouie des choses, des gens et des sens, qui est sortie toute déployée d'une anodine tasse de thé. Selon le même processus, Appelfeld remarque qu'«il suffit parfois de l'odeur d'un plat, de l'humidité des chaussures ou d'un bruit soudain pour [le] ramener au plus profond de la guerre $»^{51}$. Certes, entre les deux écrivains, le siècle a été brisé par l'Histoire et l'effet est à l'opposé : si les sensations du passé procurent au narrateur proustien un plaisir délicieux, elles replongent Appelfeld dans la guerre et son désarroi, mais l'un comme l'autre ont en commun de s'abstraire du bruit ambiant, de plonger au plus profond d'eux-mêmes pour prêter attention au ténu des sensations, ce pédoncule fragile qui nous relie à l'univers de notre passé.

38 Arrivé en Israël, loin d'être rassuré par la situation, il éprouve, ce qu'exprime son journal des années 46-50, « la nostalgie des jours où j'étais seul, entouré d'arbres et de prairies, vivant dans mon mutisme $\aleph^{52}$. Au contraire de la plupart qui témoignent au lendemain de la guerre, lui cherche le silence comme moyen de renouer avec lui-même. Son premier souci est de se retrouver :

Les premiers mots de ma main furent des appels désespérés pour trouver le silence qui m'avait entouré pendant la guerre et pour le faire revenir vers moi. Avec le même sens que celui des aveugles, j'ai compris que dans ce silence était cachée mon âme et que, si je parvenais à le ressusciter, peut-être que la parole juste me reviendrait ${ }^{53}$.

39 "Avec le même sens que celui des aveugles » : ce rapprochement vient ici donner du sens à l'intérêt que porte le narrateur, à de nombreuses reprises au cours de son récit, à des enfants aveugles ou sourds-muets. Ce sont par exemple les enfants aveugles qui 
chantent en partant pour la déportation ${ }^{54}$, ou Karl, le sourd-muet qui protège les enfants également sourds-muets de son institution, avec un courage instinctif ${ }^{55}$. Comme le narrateur, ces individus qui ont un rapport au monde empêché sont tournés vers leur vie intérieure, à l'écoute des sensations pour se repérer. Et, d'une certaine manière, à ne pas verbaliser ou identifier ce qui lui arrivait, il a lui-même vécu en aveugle :

La guerre s'était terrée dans mon corps, pas dans ma mémoire. Je n'inventais pas, je faisais surgir des profondeurs de mon corps des sensations et des pensées absorbées en aveugle ${ }^{56}$.

\section{La mémoire des langues}

Plus dramatique que l'absence de souvenirs verbalisables, plus destructeur, est le problème de la langue maternelle pour ce jeune garçon au lendemain de la guerre. Lui qui vivait au milieu de multiples langues, le roumain et le ruthène de son pays, le yiddish de ses grands-parents, l'allemand qu'il parlait avec ses parents, lorsqu'il arrive en Israël, enfin sauvé de la guerre, il se trouve contraint d'apprendre l'hébreu. Plus grave, dans ce nouveau pays tourné vers son édification, il est désormais répréhensible de parler sa langue maternelle, on lui impose donc de l'oublier, ce qui décuple sa solitude et son désarroi :

Les mots étaient les cris étouffés d'un adolescent de quatorze ans, une sorte d'aphasique qui avait perdu toutes les langues qu'il savait parler; le journal lui servait de jardin secret dans lequel il amoncelait ce qui subsistait de la langue maternelle ainsi que le vocabulaire qu'il venait tout juste d'acquérir ${ }^{57}$.

41 Le mot d'ordre est " oublie, prends racine, parle hébreu " ${ }^{58}$. C'est un déchirement, car sa langue allemande porte son enfance, c'est le seul lien qu'il ait pu conserver avec un passé dont tout a été détruit, c'est le seul héritage qui lui reste. Renoncer à sa langue maternelle, c'est tout à la fois s'amputer de son passé et faire mourir sa mère :

Ma langue maternelle et ma mère ne faisaient qu'un. À présent, avec l'extinction de la langue en moi, je sentais que ma mère mourait une seconde fois. C'était une désolation qui se répandait dans mon corps telle une drogue, lorsque j'étais éveillé, mais aussi lorsque je dormais. Dans mon sommeil j'errais avec des cohortes de réfugiés, tous bègues, et seuls les animaux, les chevaux, les vaches et les chiens sur les côtés de la route parlaient une langue fluide, comme si l'ordre des créatures s'était inversés9.

Pris dans ce dilemme entre l'hébreu à apprendre et l'allemand à oublier, dans cette nécessité vitale de retrouver les traces du passé dans la musique des sonorités et des sensations, il se tourne vers le yiddish. " À travers lui [le yiddish], j'aspirais à renouer avec mes grands-parents et leur maison des Carpates $»^{60}$. Pour retrouver son passé, il saute une génération et se tourne vers ses grands-parents, restés fidèles à la tradition rurale et religieuse. " C'est dans cette langue que bruissaient encore les souvenirs de la maison de mon grand-père, les visions de la guerre et ce qui m'appartenait en propre $\aleph^{61}$. Ces mots d'autrefois, il ne peut les faire surgir que dans la sphère intime du silence avec lui-même et c'est alors la mélodie du yiddish des grands-parents qui revient, le «Misstama » de sa grand-mère ${ }^{62}$, ce « mot étrange, incompréhensible » qu'il a retenu sans jamais en demander le sens. Dans cette langue déchue, oubliée, il retrouve la mélodie de ce qui fut la période heureuse de son existence et la douleur qui est la sienne. « [Le yiddish] symbolisait la diaspora, la faiblesse et le relâchement. [...] Sa condition d'orpheline résonnait avec mon statut d'orphelin $»^{63}$. Il contourne ainsi 
«l'étape allemande» de ses parents pour renouer avec une tradition qu'ils avaient abandonnée et les réinstaller dans leur histoire juive, malgré eux. constituée du langage de la mère et de ses intonations, de ces expressions non verbales, tout ce qui fait sens pour lui et qui ne se distingue pas encore des mots qu'il apprendra à disposer dans un continuum. Ainsi, ce n'est pas seulement par les mots, par la mémoire consciente que le narrateur cherche à retrouver sa mère, mais c'est par la "langue de l'oubli ", langue que nous oublions adultes, dès lors qu'il nous faut communiquer par un langage abstrait et conventionnel, mais qui a façonné notre rapport au monde, soupirs, rythmes, regards, gestes de don ou de retrait. Cette mère qui ouvre le récit, est ce qui l'ouvre au monde, elle qu'il revoit chaque fois dans la même position, accoudée à la fenêtre, ce que l'on peut lire ainsi : dans la maison auprès de lui, mais tournée vers les beautés du monde extérieur vers lequel elle le guide ou qu'elle ramène à l'intérieur, comme ce panier d'osier plein de fruits sauvages. C'est d'ailleurs dans cet entre-deux, entre le monde extérieur de la rue et le monde intérieur de la maison que lui apparaît sa mère, au cours de ces visions où il croit à 
[...] sa résurrection. Chaque fois que je suis heureux ou attristé, son visage m'apparaît, et elle, appuyée à l'embrasure de la fenêtre, semble sur le point de venir vers moi $^{67}$.

\section{L'invention d'un genre : témoigner entre prose et poésie}

Comment donner forme et consistance à ces «héritages aveugles»? Je voudrais montrer que cela est rendu possible par l'invention d'un genre qui consiste à témoigner entre prose et poésie, à témoigner par l'introduction de principes poétiques dans la prose (ce qui ne revient pas à de la prose poétique).

\section{Les obstacles au témoignage}

51 Au lendemain de la guerre, quand il s'essaie à raconter, Aharon Appelfeld commence par rencontrer un certain nombre d'obstacles. Je passerai rapidement sur le premier type d'obstacles. Il concerne les problèmes de la représentation ou plutôt de «l'irreprésentabilité » d'un événement extrême comme la Shoah sur lesquels Jacques Rancière fait le point ${ }^{71}$.

Notons comme précaution liminaire que, dans un retournement réflexif sur les conditions de possibilité du témoignage, Appelfeld en évoque le cas limite : quand la terreur a été trop puissante, elle a provoqué l'aphasie du survivant ${ }^{72}$. C'est le cas de ces enfants de l'enclos «Keffer » qui échappent à une mort terrible, celle de la dévoration par les chiens, mais qui en restent muets :

Les enfants avaient été sauvés, mais leur langue avait été mutilée. [...] Un ahurissement effrayant régnait dans leurs yeux. Ils restaient assis sur leurs lits la majeure partie de la journée, figés dans le silence ${ }^{73}$.

Et j'en viens aux obstacles spécifiques qu'il rencontre et qui tiennent à la fois à la particularité de son histoire et à la situation de la réception au lendemain de la guerre. En même temps que la difficulté à retrouver la trace qui relie son passé européen à sa nouvelle vie, il se heurte à l'obstacle du choix du genre : réalisme? poème? chronique? Ses premiers essais sont infructueux. Il commence par écrire des poèmes, mais ils se ramènent à des «plaintes ininterrompues d'un animal abandonné, d'une monotonie 
épuisante $\aleph^{74}$. Puis il s'essaie au témoignage. On attend de lui, comme des autres, qu'il témoigne selon une norme en vigueur, celle de la véracité du réalisme ${ }^{75}$, et selon la forme alors en usage, celle de la chronique. Or, outre les faits établis, la chronique requiert un ordonnancement chronologique. C'est précisément ce dont manque son expérience d'enfant: il ne dispose pas de faits, sa mémoire est celle du corps, des gestes, elle a accumulé des sensations qu'il n'a pu identifier, encore moins classer et situer dans le temps, il est gêné par une mémoire tout à la fois encombrée et incommunicable $e^{76}:$ «Moi, je n'avais même pas de témoignage à offrir $»^{77}$.

Le recul permet d'ailleurs de constater que les textes qui ont marqué le public sont ceux qui ont échappé à un réalisme plat, soit qu'ils soient particulièrement élaborés comme L'Espèce humaine ${ }^{78}$ de Robert Antelme dont Georges Perec dit :

Robert Antelme élabore et transforme - en les intégrant dans un cadre littéraire spécifique, alors que les autres récits concentrationnaires utilisaient des cadres romanesques élémentaires à peine diversifiés - (élabore et transforme donc) les faits, les thèmes, les conditions de sa déportation ${ }^{79}$.

soit que, comme dans Si c'est un homme ${ }^{80}$, Primo Levi réécrive des mythes et des épopées ${ }^{81}$ d'un monde littéraire partagé, soit que le témoignage soit indirect et passe par la fiction comme $W$ ou le Souvenir d'enfance ${ }^{82}$ de Georges Perec, soit encore qu'il se tourne vers la poésie, comme Paul Celan qui « déplace vers le territoire de la page blanche » et qui « désarticule l'écriture »"

En ce souci, il est accompagné par son ami l'écrivain Leïb Rochman qui, pénétré de son passé yiddish et de "la conviction qu'après l'Anéantissement on ne peut plus penser, sentir, ne parlons même pas d'écrire comme on le faisait auparavant ${ }^{84}$, refuse d'utiliser les formes conventionnelles et recherche une expression nouvelle.

Il lui faut donc trouver une forme qui ne soit ni compte-rendu réaliste ni poème ${ }^{85}$. Cette forme, - c'est l'hypothèse que je voudrais avancer - c'est celle d'un genre qu'il invente, celui d'un "témoignage poétique", d'un témoignage qui traite la prose "comme si c'était de la poésie $\aleph^{86}$, en mesurant combien le fait d'associer la poésie et le témoignage (genre qui s'étaie de documents et de faits concrets) peut relever de l'impossible.

\section{Témoigner en poète}

Tout d'abord, pour contourner les attentes, il se défend d'écrire sur la Shoah, récuse les catégories et les genres préétablis et s'en tient à écrire sur ce qu'il a vécu : "J'ai été un enfant pendant la guerre [...]. C'est à partir de ces sensations que je brode la légende $»^{87}$.

Ensuite, sa faiblesse - ses sensations minuscules -, il les retourne en écriture :

Moi, je n'avais même pas de témoignage à offrir. Je ne me souvenais pas des noms de personnes ni de lieux, mais d'une obscurité, de bruits, de gestes. C'est uniquement avec le temps que j'ai compris que ces matières premières étaient la moelle de la littérature et que, partant de là, il était possible de donner forme à une légende intime ${ }^{88}$.

61 Et dans cette perspective, son souci d'écrivain est de trouver, plus que les mots justes, le rythme juste : «Si [les] propos [de l'écrivain] ont un sens, c'est qu'il est fidèle à luimême, à sa voix, à son rythme ${ }^{89}{ }^{. . .}$et ajoutons : c'est qu'il est fidèle à ses silences, à la respiration du silence ${ }^{90}$.

Du livre A pas aveugles de par le monde, dont il admire le travail, il dit : 
C'est une expédition pour découvrir des mots, des phrases, des rythmes, qui puissent épuiser le contenu d'une âme jusque dans ses plus petits détails ${ }^{91}$.

63 À ce souci du rythme, de la musique - et du silence qui accompagne cette musique -, la forme qui répond le plus puissamment est la poésie, ou plutôt, certaines caractéristiques de la poésie, celles qui introduisent du blanc dans le texte, de la scansion, des reprises de motifs et un certain mystère, lorsque la poésie, consciente de sa gravité, se fait « musique religieuse » :

La littérature est la musique religieuse que nous avons perdue. La littérature contient toutes les composantes de la foi : le sérieux, l'intériorité, la musique, et le contact avec les contenus enfouis dans l'âme ${ }^{92}$.

Pour « tenir » cette musique, il doit refuser une mémoire envahissante qui brouille les choses, une parole qui, d'être «écoulement plat, chronologique et extérieur, sans flamme intérieure ${ }^{93}$, ne permet pas de faire advenir l'essentiel : l'émotion, la scène, le regard.

\section{«Blanchir Histoire d'une vie comme si c'était de la poésie »}

C'est pourquoi en écho au silence qui l'a accompagné, il donne dans son écriture une large place au blanc. Le blanc permet à l'écrivain qu'il est de contourner l'impasse d'une continuité narrative et logique qu'il ne peut restituer. Il ouvre la possibilité de faire émerger le réservoir de sa mémoire empli de sensations. Plus encore, dans tout texte et plus précisément dans celui de Aharon Appelfeld, le silence a une «fonction poétique » au sens étymologique du terme : il invente, il introduit des pauses, crée des ouvertures, laisse place à l'imagination, donne à interpréter. Il attire l'attention sur les échos, fait résonner ensemble les motifs, les mots et les scènes repris d'un bout à l'autre $\mathrm{du}$ récit (les arbres, l'écoulement de l'eau, les compagnies taciturnes, ou encore, plus symboliquement, le motif du seuil, etc.), il permet au lecteur d'entendre la petite musique du texte ${ }^{94}$.

Ce silence à qui l'écrivain de Histoire d'une vie donne un rôle, figure à plusieurs niveaux. Présent au niveau thématique, nous l'avons vu - tantôt allié et tantôt ennemi -, il est également présent dans la structure même des chapitres, dans leur discontinuité et, de façon plus générale et plus diffuse, dans le style.

Sur le plan de la structure, les chapitres sont nombreux (on compte trente chapitres) et plus étonnant, ils sont brefs, plus de la moitié d'entre eux comptent moins de quatre pages $^{95}$. Ils ne sont pas placés dans une succession chronologique, mais présentent de nombreux retours en arrière, des plongées dans le drame de la guerre ou dans la période heureuse qui l'a précédée, selon un rythme qui rend compte de l'intermittence de la mémoire, d'une logique intime ${ }^{96}$.

Tels des poèmes en prose, ils constituent autant de développements autonomes et denses : ils sont rarement narratifs, chaque chapitre est centré sur un événement, un thème, une sensation, un souvenir d'enfant qu'il développe sans autoriser un glissement vers d'autres considérations. Ainsi, le chapitre fait sortir du noir un événement, un objet, une personne sur quelques pages, puis le laisse s'y engloutir du seul fait qu'il ne conclut pas et que le chapitre suivant, en portant l'éclairage sur une autre zone, crée juxtaposition et discontinuité. Dans ce récit à événements en suspens, c'est l'imagination du lecteur qui vient combler les manques. Par exemple, le chapitre 6 
qui traite des enfants aveugles se termine sur l'indication livrée sans commentaire: " avant d'être poussés vers les wagons " ${ }^{97}$.

Outre qu'il figure au niveau thématique et structural, le silence figure également dans le style, ou plutôt dans le choix d'un style dépouillé. Un style dépouillé, c'est-à-dire un style qui évite des explications, des commentaires, des descriptions, des analyses psychologiques, des portraits (ou qui les limite à quelques traits rapides, tel le portrait de Maria qui n'arrive qu'à la fin du chapitre 8), ou encore un style qui évite radicalement toute couleur locale, bien que le personnage soit ballotté du centre de l'Europe jusqu'aux côtes italiennes et de là, en Israël.

Discontinuité dans la chronologie, parataxe des chapitres, économie de mots, absence de description réaliste des choses et des gens, écriture qui se refuse au déploiement de l'explication au profit de la suggestion, comme autant de blancs dans le texte. Et par ailleurs, reprise en écho de thèmes, de formules, de paysages (par exemple, les arbres, les champs de maïs, les cours d'eau...). Tout cela relève d'une écriture poétique qui invite davantage à la contemplation, à l'écoute de sensations diffuses qu'à la vérification réaliste :

Un instant de contemplation [...] vous remplit de couleurs, de sons et de rythmes

[...] Une vraie contemplation, comme la musique est dénuée de contenu matériel ${ }^{98}$.

71 Mutatis mutandis, parce que, pour Aharon Appelfeld, il ne s'agit pas seulement d'inventer une forme, mais de rendre compte des dégâts de l'Histoire sur une vie - et sur celle d'un peuple "arrêté par une gigantesque main satanique ${ }^{99}-$, cela n'est pas sans rappeler, certes de très loin, celui qui a inventé le poème en prose sans le savoir, Aloysius Bertrand. Au rebours de la profusion verbale romantique du XIx siècle, à propos des textes - suggestifs, étranges, surgis de la nuit et y retournant--, de son Gaspard de la nuit, il recommande de «blanchir comme si c'était de la poésie ${ }^{100}$. De même, par sa façon d'introduire du blanc entre les chapitres, de faire apparaître et disparaître une scène, de pratiquer l'asyndète, la juxtaposition, la simplicité énigmatique, Appelfeld invente un témoignage qui n'est ni réaliste, ni fictionnel, mais qui est résonance poétique, qui tresse la parole avec le silence dont elle procède et qui s'étend après elle, qui donne un timbre au silence, ici angoissé, là apaisé, ici clair et libre et là, assombri et étouffé.

Ainsi, le silence que l'écrivain introduit dans son texte répond aux silences de sa propre histoire de personnage solitaire et occupe une place efficiente. De ce fait, il requiert de son lecteur une écoute attentive, une "hospitalité » ${ }^{101}$ qui réaliserait, dans la lecture, celle qui a tant manqué à l'enfant-loup qu'il a été.

73 Paradoxe d'un témoignage qui serait poétique, invention d'une poétique du silence donc, dont on peut se demander si elle n'est pas paradoxalement issue de ses premières tentatives infructueuses d'écriture. On se souvient que Aharon Appelfeld commence par essayer d'écrire selon des genres opposés, des poèmes et une chronique ${ }^{102}$ et qu'il les abandonna parce que l'un comme l'autre sonnait faux. On peut se demander si, avec Histoire d'une vie, ce n'est pas en frayant une voie inusitée entre ces deux genres inconciliables, qu'il a inventé ce genre oxymorique du «témoignage poétique » qui " com-pose » la rêverie poétique avec le document historiographique de la chronique.

Car, de la chronique, il a gardé le récit qui, en se calant sur de larges périodes ${ }^{103}$, échappe à une chronologie. À la poésie, il a emprunté les scènes détachées les unes des autres, sans fil de continuité entre elles, le rythme serré des phrases, la narration brève 
d'une scène, le détail saisi sur le vif, les visions, qui sont autant de morceaux détachés du temps, de récits poétiques suspensifs.

Cette forme née d'un métissage des genres, il faut souligner quel retournement elle réussit à opérer afin de promouvoir le silence : si, dans le poème, du fait du blanc qui entoure les strophes, le silence a présence reconnue, en revanche, dans la prose, il est généralement déterminé négativement comme un faire-valoir de la parole, un espace à occuper au profit du verbe. Dans Histoire d'une vie, ce sont les silences qui viennent travailler la prose, en se tenant au plus près de la subjectivité ${ }^{104}$.

À considérer la genèse de ce genre qui s'invente au moyen d'un croisement, le rapprochement s'impose une nouvelle fois avec La recherche du temps perdu: ce vaste roman de la mémoire est issu d'une voie qui se trace entre deux genres antithétiques, la narration et le commentaire, entre un « je » qui serait trop effusif et un « il » qui serait trop distant ${ }^{105}$. Comme Marcel Proust, Aharon Appelfeld invente une forme qui traduit les «intermittences du cœur»-pour reprendre le titre premier de La recherche-, comme lui, il commence par une succession de souvenirs d'enfance, le voyage dans la famille, le panier de fraises, les promenades et comme lui, il cherche à approcher la vérité d'une sensation par une formulation poétique qui s'invente, pour le premier, par la rhétorique poétique avec les anneaux de la métaphore et pour le deuxième, par les silences de la réticence. Comme Proust, également, qui circule entre l'anecdote singulière et l'énoncé des lois générales, Appelfeld passe de la subjectivité de ses sensations à l'anonymat d'une vie que son titre suggère. Comme Proust, enfin, il découvre à la fin de son livre, non pas sa vocation, mais son style d'écrivain qui lui permet d'écrire le livre que le lecteur vient de lire...

Pour tenter de répondre à la question liminaire, comment trois enfants plongés dans la guerre, qui ont subi dans la solitude trois expériences différentes, peuvent-ils témoigner, on peut avancer que chacun d'eux a inventé par la littérature une solution singulière. Ne pouvant témoigner selon un mode établi, ils sont allés chercher, au-delà des genres, une forme qui pourra dire la souffrance dans leur corps. Et c'est l'écriture blanche pour Georges Perec dont l'enfance fut amputée par «l'Histoire avec sa grande hache ", la phrase unique à courts-circuits pour Raymond Federman captant depuis le débarras sombre où sa mère l'a caché les pleurs et les cris lors de la rafle qui emporte ses parents et enfin, la poétique du silence pour Aharon Appelfeld qui a conservé en lui la musique des jours anciens. Mais comme il est difficile de conclure sur le silence autrement qu'en faisant silence soi-même, je laisserai le dernier mot à Edmond Jabès, poète du blanc, de la marge, du silence :

Le livre est le lieu où l'écrivain fait, au silence, le sacrifice de sa voix.

Ainsi toute marge est plage d'un aveu contenu, au bord de laquelle les vocables revenus scellent, avec la mer, leur alliance ${ }^{106}$. 


\section{BIBLIOGRAPHIE}

AMÉRY, Jean (1995). Par-delà le crime et le châtiment - Essai pour surmonter l'insurmontable, traduit de l'allemand par Françoise Wuilmart, Arles, Actes Sud. [1 $1^{\text {re }}$ publication : 1966].

ANTELME, Robert (1957). L'Espèce humaine, Paris, Gallimard. [1 19e édition : 1947].

APPELFELD, Aharon (2004). Histoire d'une vie, traduit de l'hébreu par Valérie Zenatti, Paris, Éditions de L'Olivier ; Paris, Seuil, coll. « Points », 2005. [1 $1^{\text {re }}$ publication : Sippur hayyim, Jérusalem, Keter, 1999].

APPELFELD, Aharon (2006). L'héritage nu, traduit de l'anglais par Michel Gribinski, Paris, Éditions de L'Olivier. [1 ${ }^{\text {re }}$ publication : (1994) Beyond Despair].

APPELFELD, Aharon (2012). «Frère d'âme ", Préface à Leïb Rochman, À pas aveugles de par le monde, Paris, Denoël. [1 $1^{\text {re }}$ publication : 1979].

ARENDT, Hannah (1972). Les Origines du totalitarisme - Le système totalitaire, Paris, Seuil. [1 ${ }^{\text {re }}$ publication : 1951].

ARENDT, Hannah (2008). Idéologie et terreur, trad. Marc de Launay, Introduction et notes de Pierre Bouretz, Paris, Hermann, coll. « Le Bel aujourd'hui ». [Rédaction : 1952].

ARENDT, Hannah (1981 et 1983). La Vie de l'esprit, volume I : La Pensée, volume II : Le Vouloir, trad. L. Lotringer, Paris, PUF, coll. «Philosophie aujourd'hui ».

AUSTER, Paul (1999). « Conversation avec Edmond Jabès », trad. Angélique Lévi, in Steven Jaron (dir.), Portrait(s) d'Edmond Jabès, Paris, Bibliothèque nationale de France.

BANON, David (1987). La lecture infinie. Les voies de l'interprétation midrachique, Paris, Seuil. BERTRAND, Aloysius (1980). Gaspard de la nuit, Paris, Poésie/Gallimard. [1 ${ }^{\text {re }}$ publication : 1842]. CELAN, Paul (1998). « Grille de Paroles » in Choix de poèmes réunis par l'auteur, édition bilingue, traduction, présentation et notes de Jean-Pierre Lefebvre, Paris, Poésie/Gallimard. [Rédaction de «Strette » : 1958].

DERRIDA, Jacques (1972). «La pharmacie de Platon » in La dissémination, Paris, Seuil.

[1 $1^{\text {re }}$ publication : 1968].

FEDERMAN, Raymond (2002). La Voix dans le débarras, The voice in the closet, Paris, Les Impressions nouvelles.

HASSOUN, Jacques (1993). L'exil de la langue. Fragments de langue maternelle, Paris, Points hors ligne. ITZHAKI, Masha (2003-2004). « Aharon Appelfeld, Histoire d'une vie : autobiographie d'une écriture ", Yod nº 9.

ITZHAKI, Masha (2010). Aharon Appelfeld : le réel et l'imaginaire, Paris, L'Harmattan.

JABÈS, Edmond (1988). Le Livre des Questions, Tome I, Paris, Gallimard, coll. « L'Imaginaire ». JABÈS, Edmond (1989). « Elya », in Le Livre des Questions, Tome II, Paris, Gallimard, coll. « L'Imaginaire ». JABÈs, Edmond (1991). Le Livre de l'hospitalité, Paris, Gallimard. KofMAN, Sarah (1986). Paroles suffoquées, Paris, Galilée. 
LEVI, Primo (2006). Si c'est un homme, traduit de l'italien par Martine Schruoffeneger, Paris, Julliard. [Rédaction : $1947 ; 1^{\text {res }}$ publications : Turin, 1958-1976 ; $1^{\text {re }}$ trad. française : Paris, 1987]. PEREC, Georges (1975). W ou le souvenir d'enfance, Paris, Denoël.

PEREC, Georges (1992). L.G., une aventure des années soixante, Paris, Seuil, coll. « La Librairie du Xx siècle ».

PROUST, Marcel (1954). Contre Sainte-Beuve, Paris, Gallimard. [Période de rédaction : 1908-1910]. PROUST, Marcel (1987). « Combray », in A la recherche du temps perdu, dir. Jean-Yves Tadié, Paris, Gallimard Pléiade.

RANCIÈRE, Jacques (2003). Le destin des images, Paris, La Fabrique éditions.

RIMBAUD, Arthur (1999), Poésie. Une saison en enfer, Illuminations, Paris, Poésie/Gallimard [1873].

ROCHMAN, Leïb (2012). À pas aveugles de par le monde, traduit de l'hébreu par Rachel Ertel, Paris, Denoël. Préface de Aharon Appelfeld.

SABBAH, Danièle (2011). « Au milieu du chaos, la poésie (sur Primo Levi) », in L'Exil et la différence, Eidôlon $\mathrm{n}^{\circ}$ 90, Bordeaux, PUB.

\section{NOTES}

1. Edmond Jabès (1988), p. 72.

2. Georges Perec (1975), p. 17

3. De l'autobiographie, il n'a ni la chronologie, ni la continuité, ni le réalisme minimal attendu (dates, lieux, personnages, paysages, etc.). L'en éloigne également sa présentation sous forme de chapitres distincts et numérotés, centrés chacun sur un «thème » spécifique, comme nous le verrons en fin de développement.

4. Arthur Rimbaud (1999), Lettre à Paul Demeny, 15 mai 1871, "Les inventions d'inconnu réclament des formes nouvelles ».

5. Ainsi que le veut la conception chrétienne de l'incarnation de Jésus-Christ, comme Verbe qui s'est fait chair.

6. Etymologie latine de « enfant ", mot à mot : « celui qui ne parle pas ».

7. Jacques Derrida (1972).

8. A. Appelfeld (2004), p. 15.

9. Ibid., p. 19-20.

10. Ibid., p. 20.

11. Ibid., p. 21.

12. Ibid.

13. Ibid., p. 31

14. Ibid.

15. Ibid., p. 121.

16. Cf. p. 82

17. A. Appelfeld (2004), p. 166-167.

18. La distinction que Hannah Arendt développe entre « monos » («solitary man ») et « eremos » (« lonely man »), H. Arendt (2008).

Voir également H. Arendt (1981 et 1983).

19. M. Proust (1987), « Combray », p. 36-37.

20. A. Appelfeld (2004), p. 67-68.

21. Ibid., p. 69. 
22. Ibid., p. 80.

23. Ibid., p. 47.

24. Ibid., p. 47.

25. Ibid., p. 25

26. Ibid., p. 25.

27. Ibid., p. 22.

28. Ibid. p. 122.

29. Pour reprendre le titre de Sarah Kofman (1986).

30. A. Appelfeld (2004), p. 147.

31. Ibid., p. 70.

32. Ibid., p. 75.

33. J. Améry (1995), voir "Dans quelle mesure a-t-on besoin de sa terre natale?», particulièrement p. 112 sqq.

34. A. Appelfeld (2004), p. 75.

35. Ibid., p. 80-83.

36. Ibid., p. 81-82.

37. Kierkegaard.

38. A. Appelfeld (2004), préface, p. 8.

39. Ibid., p. 9.

40. Ibid., p. 166.

41. Ibid., p. 123.

42. Ibid., p. 122.

43. Pour une mise en œuvre «stylistique » de ce court-circuit, voir Raymond Federman (2002).

44. A. Appelfeld (2004), p. 109-110.

45. Ibid., p. 9.

46. Ibid., p. 109.

47. Ibid., p. 110.

48. Ibid., p. 111.

49. Ibid., p. 111.

50. M. Proust (1987), « Combray », p. 47.

51. A. Appelfeld (2004), p. 110.

52. Ibid., p. 147.

53. Ibid., p. 127.

54. Ibid., p. 56 sqq.

55. Ibid., p. 87.

56. Ibid., p. 223.

57. Ibid., p. 130.

58. Ibid., p. 167.

59. Ibid., p. 133.

60. Ibid., p. 179.

61. Ibid., p. 175.

62. Ibid., p. 15.

63. Ibid., p. 176.

64. Ibid., p. 13.

65. Ibid., p. 138.

66. Jacques Hassoun (1993), p. 62.

67. A. Appelfeld (2004), p. 68.

68. Ibid., p. 80-83.

69. Ibid., p. 139.

70. Ibid., p. 185. 
71. Jacques Rancière (2003). Jacques Rancière rappelle que ces problèmes de représentation relèvent de deux ordres hétérogènes, d'une part l'ordre religieux tenant à l'interdit de la représentation ou aux limites éthiques que l'on se donne, d'autre part les questions tenant aux limites mêmes de l'acte de représenter (la mimesis, la fiction, etc.), questions qui se posent en tous temps, mais qui là se trouvent exacerbées du fait qu'il s'agit d'événements extrêmes.

72. H. Arendt (1972), p. 177-178. Hannah Arendt consciente de la difficulté de témoigner, parle de ces victimes pétrifiées, lorsqu'elle évoque « la terreur animale sans espoir - celle qui en face de l'horreur réelle, présente, paralyse inexorablement tout ce qui n'est pas pure réaction ».

73. A. Appelfeld (2004), p. 93.

74. Ibid., p. 136. À ce sujet, voir Masha Itzhaki (2011).

75. Les témoignages « réalistes" répondent à deux critères: d'une part, ils s'appuient sur des documents, une accumulation de détails, d'autre part, ils s'organisent en chronique, en récit ordonné et reposant sur des données identifiables, communicables, voire authentifiées.

76. A. Appelfeld (2004), p. 148-149.

77. Ibid., p. 128.

78. Robert Antelme (1957).

79. G. Perec (1992), p. 94. La suite de la citation est : « ...Et d'abord, il choisit de refuser tout appel au spectaculaire, d'empêcher toute émotion immédiate, à laquelle il serait trop simple, pour le lecteur, de s'arrêter. "

80. Primo Levi (2006).

81. La Bible, L'Odyssée, L'Enfer de Dante sont parmi les textes qui en nourrissent l'intertextualité, voir D. Sabbah (2011).

82. G. Perec (1975).

83. Paul Celan (1998), «Strette», p. 154.

« Dé-placé dans

le territoire

à la trace non-trompeuse :

herbe écriture désarticulée »

84. A. Appelfeld, préface à Leïb Rochman (2012), p. 10-11.

85. A. Appelfeld (2006), p. 12 : «Mes premières tentatives d'écrire furent des poèmes et une chronique. Les premiers poèmes n'étaient rien de plus que des hurlements étouffés, des appels au secours, des cris adressés à Dieu. »

86. Aloysius Bertrand (1842), p. 301.

87. A. Appelfeld (2004), p. 150.

88. Ibid., p. 128.

89. Ibid., p. 150.

90. Car son matériau étant les sensations, il s'agit de rester au plus près du corps et donc "d'écrire comme on respire ». Le poète Edmond Jabès décrit ce phénomène, à propos de la forme «blanche» par excellence, la forme aphoristique: "Dans mon cas, l'aphorisme - que l'on pourrait appeler la phrase nue - vient du besoin d'entourer les mots de blancheur afin de les laisser respirer. Je souffre d'asthme, comme vous le savez, et j'ai parfois du mal à respirer. En donnant du souffle à mes mots, j'ai souvent l'impression que j'améliore ma respiration. C'est incroyable à quel point votre écriture est en symbiose avec votre corps. [...] ... nous travaillons avec nos corps, notre souffle, notre rythme et [...] l'écriture, en quelque sorte, mime tout cela. L'écriture œuvre dans deux directions. Elle est à la fois expansion et contraction. [...] Ce n'est que bien plus tard que je compris que ces termes ne s'excluaient pas, et même que le style n'est rien d'autre que cela, c'est l'essence même de l'écriture. Il faut écrire comme on respire » Paul Auster (1999), p. 86-87.

91. A. Appelfeld, préface à L. Rochman (2012), p. 12-13.

92. A. Appelfeld (2004), p. 140. 
93. Ibid., p. 215.

94. David Banon (1987) le souligne: «Le blanc est comme le lieu d'une réserve de sens que le texte recèle. Il permet les ouvertures par où se faufilent les transformations. Le blanc est une incitation à l'interprétation par le non-dit qu'il suggère. »

95. Dans la traduction française, du moins, les chapitres comptent quatre/cinq pages en moyenne. Seuls deux chapitres sont longs : le premier (14 pages) et le dernier (16 pages). À ce sujet, voir Masha Itzhaki (2003-2004).

96. Ce qui fait chronologie, c'est une périodisation reconstruite par le lecteur. Se dessinent quatre temps qui sont plutôt quatre «périodes mentales » articulées autour de la césure de la guerre :

. avant la guerre, période de complétude enfantine ;

- pendant la guerre, période de solitude apeurée ;

. aux lendemains de la guerre, désœuvrement et « restauration » des corps ;

. enfin, période de l'installation en Israël et de la difficile adaptation jusqu'à « nous conduire ici » (derniers mots du livre, p. 236).

97. A. Appelfeld (2004), p. 61.

98. Ibid., p. 164.

99. A. Appelfeld (2006), p. 28.

100. A. Bertrand (1842), p. 301-302. Dans ses «Instructions à Monsieur le Metteur en pages», le poète Aloysius Bertrand recommande de «Blanchir comme si c'était de la poésie ».

101. « De toi, je prends congé, mais vivrai de ta lecture.

Incommensurable est l'hospitalité du livre. »E. Jabès (1991), p. 100.

102. A. Appelfeld (2006), p. 12.

103. Voir note 97.

104. Cette écriture entre la subjectivité d'une histoire singulière et l'impersonnalité d' "une » vie, entre témoignage et poésie nous rappelle qu'il invente

- une forme : le témoignage poétique,

- un actant /une valeur : le silence,

- une subjectivité : l'histoire d'une vie parmi d'autres, représentant des milliers d'autres, qui ont été broyées par l'horreur.

105. Ce dont témoigne M. Proust (1954).

106. E. Jabès (1989), p. 199.

\section{RÉSUMÉS}

L'une des caractéristiques les plus originales de l'écrivain Aharon Appelfeld réside dans son écriture du silence. Des silences est plus exact, car selon la parole qui les fait résonner, ils offrent des tessitures multiples: silence heureux d'une mère contemplative, silence recueilli des hommes en prière, murmure intérieur de l'enfant solitaire, gorge fermée de l'enfant traqué, cinéma muet des scènes d'horreur, secret de la judaïté, aphasie du survivant ou encore épaisseur d'un silence floconneux que traverse le mot allemand pour revenir à la surface et faire surgir la mémoire.

Entre non-langage, langues à taire, langue à apprendre, langue à désapprendre, l'écriture nue d'Aharon Appelfeld se fraie une âpre voie. Le style - l'inscription, sur la page, des mots et du 
néant dont ils émergent - reconstitue une identité et donne voix à l'écrivain par-delà l'exil, la perte du pays, la perte des langues et des êtres chers qui les parlèrent.

Cette contribution se propose de mettre à jour quelques-uns des enjeux de l'écriture du silence.

One of the most original features of the writer Aharon Appelfeld is his writing of silence. Or rather of silences, since according to the words which make them resonate, they offer multiple textures : the blissful silence of a contemplative mother, the concentrated rapt silence of men in prayer, the inner whispers of a lonely child, the stuffed throat of the chased child, the silent cinema of horrific scenes, the hidden Jewishness, the aphasia of the survivor or the thick silence springing out of a German word and thus reviving past memories.

Among all these non-languages, silenced languages, languages to be learnt and languages to forget, the naked writing of Aharon Appelfeld struggles its way through. His style-the inscription of the words and of the nothingness from which they originate-reconstructs an identity and gives a voice to the writer despite the exile, the loss of the homeland, of the languages and of the loved ones who used to speak them.

This contribution shall attempt to update some of the stakes of this writing of silence.

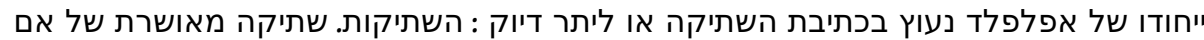

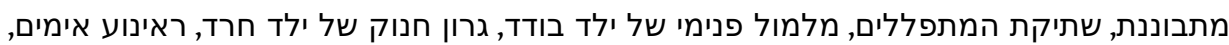
אפאסיה של ניצול, סוד היהדות, שתיקה זועקת של מילתי מילה גרמנית המית המשמשת זרז להיזיזכרות.

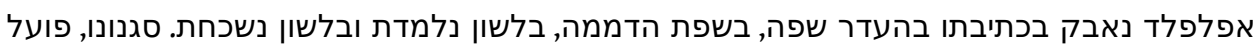

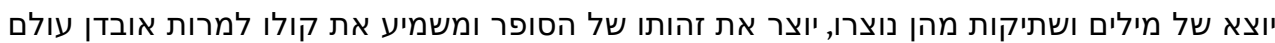

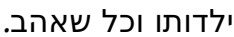

\section{INDEX}

Keywords : silence, witness, literary genres, poetics of memory, mother tongue, identity, childhood, Holocaust, Appelfeld Aharon (1932-), literature

Mots-clés : silence, témoignage, genres littéraires, poétique de la mémoire, langue maternelle, identité, enfance, Appelfeld Aharon (1932-)

מילות מפתח

דממה, שתיקות, עדות, סוגות ספרותיות, פואטיקה של זיכרון, שפת אם, זהות, ילדות:

Thèmes : littérature

Index chronologique : Shoah 